\title{
AMBIPOLAR SI NANOWIRE FIELD EFFECT TRANSISTORS FOR LOW CURRENT AND TEMPERATURE SENSING
}

\author{
D. Sacchetto, G. De Micheli, and Y. Leblebici
}

EPFL - Ecole Polytechnique Fédérale, Lausanne, Vaud, SWITZERLAND

\begin{abstract}
This paper reports on the fabrication and characterization of a pA current and temperature sensing device with ultra-low power consumption based on a Schottky barrier silicon nanowire transistor. Thermionic and trap-assisted tunneling current conduction mechanisms are identified and discussed on the base of the device sensitivity upon current and temperature biasing. In particular, very low current sensing properties are confirmed also with previously reported polysiliconchannel nanowire Schottky barrier transistors. demonstrating that these devices are suitable for temperature and current sensing applications. Moreover, the process flow compatibility for both sensing and logic applications makes these devices suitable for heterogeneous integration.
\end{abstract}

\section{KEYWORDS}

Schottky barrier, sensing, nanowire, FET, ambipolar

\section{INTRODUCTION}

The last decades have seen an exponential increase in CMOS research effort to push forward the technological limit for device density. Future technological innovations enabling ever higher circuit densities predicted by Moore's law will most likely be concentrated on novel materials, innovative device structures or different state variables other than charge [1]. Moreover, novel functionalities are attractive for sensing applications [2]. The dual advantage of density and peculiar sensing properties well combine in one dimensional structures, such as silicon nanowires.

In this work we extend the application of $\mathrm{Si}$ nanowires to current and temperature sensing. A range of device operation conditions are investigated, showing how an ambipolar device can be used for different applications, the only requirement being the biasing condition.

Section 2 describes the device concept. Then in Section 3 the fabrication method is reported. In Section 4 general current-voltage dependence of Schottky barrier FETs are presented. In Section 5 quasi-static device operation under different biasing conditions are discussed. A way to operate this device with low voltages is proposed. Then Section 5 reports on the $\mathrm{I}_{\mathrm{ds}}-\mathrm{V}_{\mathrm{gs}}$ dependence under different temperature regimes. An effective Schottky barrier height of the devices is extracted by means of an activation energy method. Finally in Section 6 current and temperature sensing applications of Schottky barrier FETs are discussed.

\section{DEVICE DESCRIPTION}

The device scheme consists of a suspended $\mathrm{Si}$ nanowire having NiSi silicide source/drain junctions with 2 parallel polysilicon gates having gate-all-around (GAA) configuration on a SOI substrate. A typical device with $20 \mu \mathrm{m}$ long Si nanowire channel is shown in Figure 1(a). Typical Si nanowire with drop-like crosssection shape has an average diameter of $100 \mathrm{~nm}$ (see Figure 1(b)).

a)

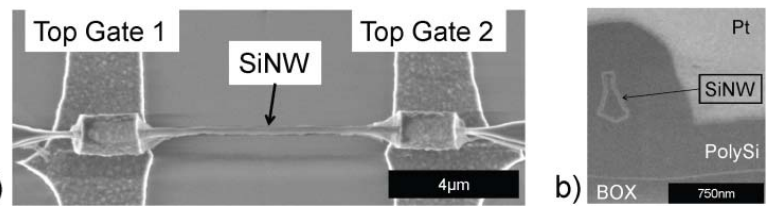

Figure 1: a) A $20 \mu \mathrm{m}$ long Si nanowire with 2 parallel GAA polysilicon gates having $4 \mu \mathrm{m}$ gate lengths. b) A FIB cut cross-section image showing the $\mathrm{Si}$ nanowire channel surrounded by a $500 \mathrm{~nm}$ polysilicon top gate.

\section{DEVICE FABRICATION}

The process flow is based on a previously reported technique utilized for the fabrication of verticallystacked ultra-dense Si nanowire FET arrays [3]. Here a deep reactive ion etching technique is adapted to form a single suspended $\mathrm{Si}$ ribbon over the buried oxide. A sacrificial oxidation is performed to reduce the crosssection down to a $100 \mathrm{~nm}$ (see Figure 1(b)). Then a 20 $\mathrm{nm}$ dry oxidation is performed followed by a $500 \mathrm{~nm}$ polysilicon layer deposited by low pressure chemical vapor deposition. Contact regions are etched in a low temperature oxide passivation layer. Then a $50 \mathrm{~nm} \mathrm{Ni}+$ $10 \mathrm{~nm} \mathrm{Al} \mathrm{bi-layer} \mathrm{is} \mathrm{evaporated} \mathrm{and} \mathrm{annealed} \mathrm{to} \mathrm{form} \mathrm{a}$ silicide/Si mid-gap Schottky junction. The Al is used as a cap layer to prevent $\mathrm{Ni}$ oxidation during the silicidation step and to provide a good interface with final metallization. Finally, $1 \mu \mathrm{m}$ thick $\mathrm{Al}$ is patterned for electrical characterizations.

\section{I-V DEPENDENCE}

For Schottky barrier FETs, $\mathrm{I}_{\mathrm{ds}}$ can be described by the sum of a tunneling $I_{\text {tunnel }}$ and a thermionic-emission $I_{\text {th }}$ component [4]:

$$
\begin{gathered}
I_{d s}=I_{\text {th }}+I_{\text {tunnel }} \\
I_{\text {tunnel }}=A_{C} \frac{q^{2} F^{2}}{8 \pi h \varphi_{\text {Beff }}} e^{\left(-\frac{8 \pi}{3 h q F} \sqrt{2 m\left(q \varphi_{\text {Beff }}\right)^{3}}\right)} \\
I_{t h}=A_{C} A^{*} T^{2} e^{\left(-\frac{\varphi_{\text {Beff }}}{k T}\right)}\left[e^{\left(-\frac{V a}{k T}\right)}-1\right]
\end{gathered}
$$


Where $A_{C}$ is the contact area of the source to channel junction, $\mathrm{F}$ the electric field across the Schottky barrier, A the Richardson's constant, $h$ the Plank's constant, $m$ the electron rest mass, $\mathrm{q}$ the elementary electron charge, $\varphi_{\text {Beff }}$ the effective Schottky barrier height, $T$ the temperature, $\mathrm{V}_{\mathrm{a}}$ the applied voltage across the Schottky junction, k the Boltzmann's constant.

\section{ELECTRICAL CHARACTERISTIC}

\section{Ambipolar behavior}

All the following measurements investigate the electrical behavior using the handle layer of the wafer as the back gate and the top gate at the source end (gate 1 in Figure 1) for a $20 \mu \mathrm{m}$ long Schottky barrier Si nanowire FET with $100 \mathrm{~nm}$ channel diameter (Figure 1(b)).

$\mathrm{A} \mathrm{V}_{\mathrm{gs}}$ sweep ranging between $-5 \mathrm{~V}$ and $+5 \mathrm{~V}$ has been performed at different $V_{b g}$ voltages. Large subthreshold slopes are expected in this kind of devices since the total current is determined by a thermionic emission and a tunneling components (equation (1-3)), giving rise to two distinct subthreshold regimes. We can indeed improve the quasi-static characteristic of the device by exploiting the back-gate to limit the conductance of one type of carrier. As suggested by Lin et al. [5] for a carbon nanotube Schottky barrier FET, a back gate voltage is capable to turn the device from an ambipolar to a unipolar behavior. As shown in Figure 2 the device has an ambipolar behavior, with unbalanced $\mathrm{p}$ - and nbranches. Since the gate length of the top gate is shorter compared to the back gate, the main effect we observe by changing $\mathrm{V}_{\mathrm{bg}}$ is a modification of the ambipolar curve, in particular a modulation of the subthreshold swing together with a strong modulation of the nbranch current by lowering the $\mathrm{I}_{\mathrm{OFF}}$ current contribution from holes injection at low $\mathrm{V}_{\mathrm{gs}}$. Nevertheless, if on the one hand the $\mathrm{V}_{\mathrm{bg}}$ can be used to improve the ambipolar $\mathrm{I}_{\mathrm{ds}}-\mathrm{V}_{\mathrm{gs}}$ curve, on the other side the lower $\mathrm{I}_{\mathrm{OFF}}$ becomes more sensitive to temperature variation, which is suitable for temperature sensing applications.

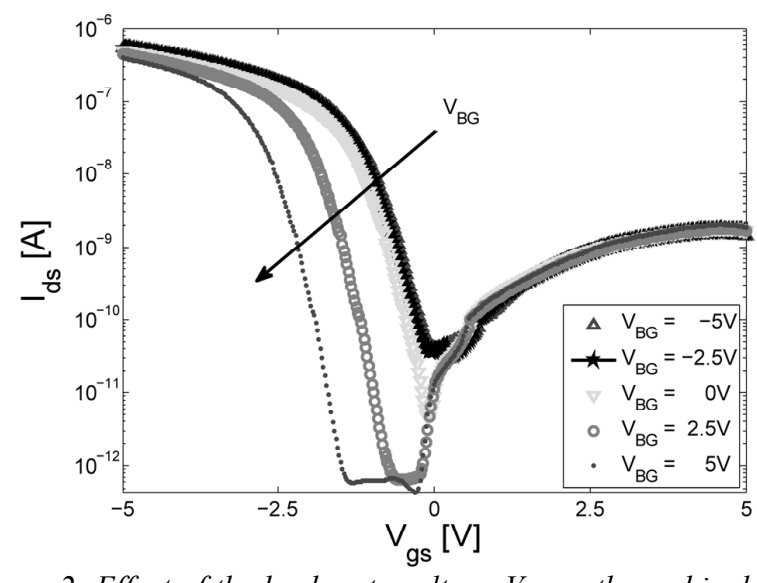

Figure 2: Effect of the back-gate voltage $V_{b g}$ on the ambipolar $I_{d s}-V_{g s .}$. The ambipolar curve is shifted toward more negative voltages for increasing $V_{b g}$. The $n$-branch swing for $V_{b g}=5 \mathrm{~V}$ is improved compared with the other cases. This is attributed to partial suppression of the holes conductance at low $V_{g s}$.

\section{Temperature effects on the electrical characteristic}

The $\mathrm{I}_{\mathrm{ds}}-\mathrm{V}_{\mathrm{gs}}$ dependence with $\mathrm{T}$ is mainly attributed to the $\mathrm{I}_{\text {th }}$, however $\mathrm{T}$ also influences the $\mathrm{I}_{\text {tunnel }}$ since hotter carriers pass through a narrower Schottky barrier, leading to an increasing current level [6]. The $\mathrm{I}_{\mathrm{OFF}}$ current is increasing exponentially with temperature and, as suggested by equation (3), its main contribution is a thermionic emission component. A different behavior has been observed for the $\mathrm{I}_{\mathrm{ON}}$ current. Increasing the temperature makes the $\mathrm{I}_{\mathrm{ON}}$ current to decrease until the temperature reaches $55^{\circ} \mathrm{C}$ and then it rise exponentially with linear increase of $\mathrm{T}$. At lower temperatures tunneling and trap-assisted tunneling are more important than thermionic emission. Rising $\mathrm{T}$ up to $70^{\circ} \mathrm{C}$ makes the charges trapped into the gate oxide to un-trap, reducing the $\mathrm{I}_{\text {tunnel }}$ component. A different behavior is observed for the $\mathrm{I}_{\mathrm{ON}}$ currents for $70^{\circ} \mathrm{C} \leq T \leq 115^{\circ} \mathrm{C}$. In this range, the $\mathrm{I}_{\mathrm{ON}}$ exponentially increases with $\mathrm{T}$. This effect is evidence of two main current components, for which the $\mathrm{I}_{\mathrm{ON}}$ changes from a tunneling to a thermionic emission dominated regime.

A set of $\mathrm{I}_{\mathrm{ds}}-\mathrm{V}_{\mathrm{gs}}$ curves (Figure 3) taken at different temperatures at constant $\mathrm{V}_{\mathrm{ds}}=100 \mathrm{mV}$ and $\mathrm{V}_{\mathrm{bg}}=5 \mathrm{~V}$ are used to extrapolate the Arrhenius plot shown in Figure 5. The constant $\mathrm{V}_{\mathrm{bg}}=5 \mathrm{~V}$ is used to set the device operation more favorable for electron conductance at low $\mathrm{V}_{\mathrm{gs}}$. Constant subthreshold swings $\approx 110 \mathrm{mV} / \mathrm{dec}$ are observed independently from the temperature (see Figure 4). Low negative $V_{\text {gs }}$ voltages ranging from $-1 \mathrm{~V}$ to $0 \mathrm{~V}$ show an almost linear slope with inverse of temperature and can be correlated to a thermionic-emission regime. However, for this $\mathrm{V}_{\mathrm{gs}}$ range the current level is on the order of fAs, which is comparable to the background noise, and it cannot be used to extrapolate the Schottky barrier height. Another distinct regime is observed for $-0.3 \mathrm{~V} \leq \mathrm{V}_{\mathrm{gs}} \leq-0.5 \mathrm{~V}$, for which the slopes are greatly affected by tunneling. This regime shows a dominant tunneling component for the two lowest temperatures. Finally, an exponential dependence with $\mathrm{T}$ is observed again for $\mathrm{V}_{\mathrm{gs}} \geq 0 \mathrm{~V}$ with the exception of the lower temperature. All these regimes demonstrate that the current in our device is mainly thermionic for $\mathrm{T} \geq 70^{\circ} \mathrm{C}$ and that the tunneling contribution is trap assisted.

The slopes from the Arrhenius plot are then used to extract the effective Schottky barrier height $\varphi_{\text {Beff }}$ with the activation energy $E_{a}$ method. As shown in inset $A$ of Figure 6 , an average effective barrier height $\mathrm{E}_{\mathrm{a}} \approx 450 \pm 5$ $\mathrm{meV}$ is found over a large range of $\mathrm{V}_{\mathrm{gs}} \geq 0.2 \mathrm{~V}$. However, these values cannot be taken as Schottky barrier height since in this regime the device has both tunneling and thermionic components. As suggested by Svensson et al. [7], a better evaluation of the Schottky barrier height can be taken at the maximum of $E_{a}$ for low current levels. As shown in the inset B of Figure 6, this maximum corresponds to $\mathrm{V}_{\mathrm{gs}}=-0.45 \mathrm{~V}$ and gives a $\varphi_{\text {Beff }}$ $=525 \mathrm{meV}$, confirming the mid-gap Schottky barrier height. 


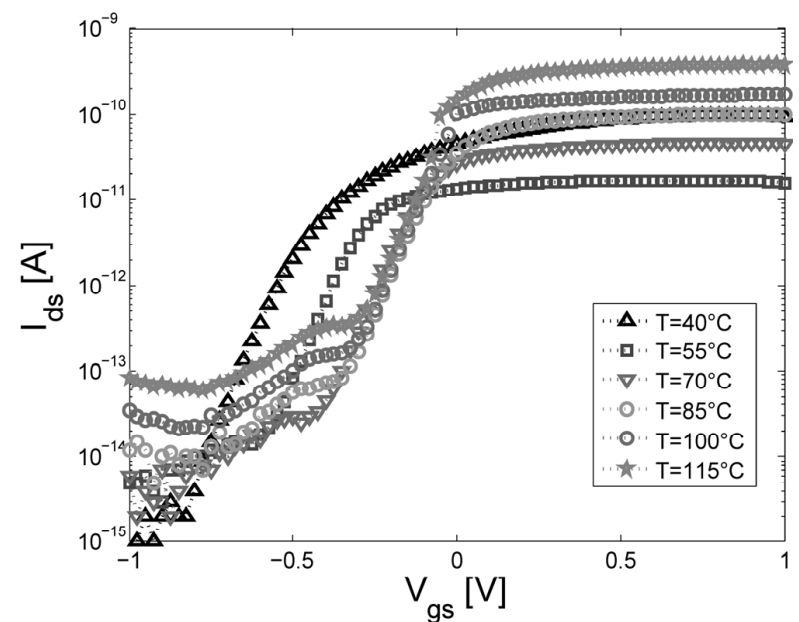

Figure 3: Effect of the temperature on the ambipolar $I_{d s}-V_{g s}$ at $V_{d s}=100 \mathrm{mV}$.

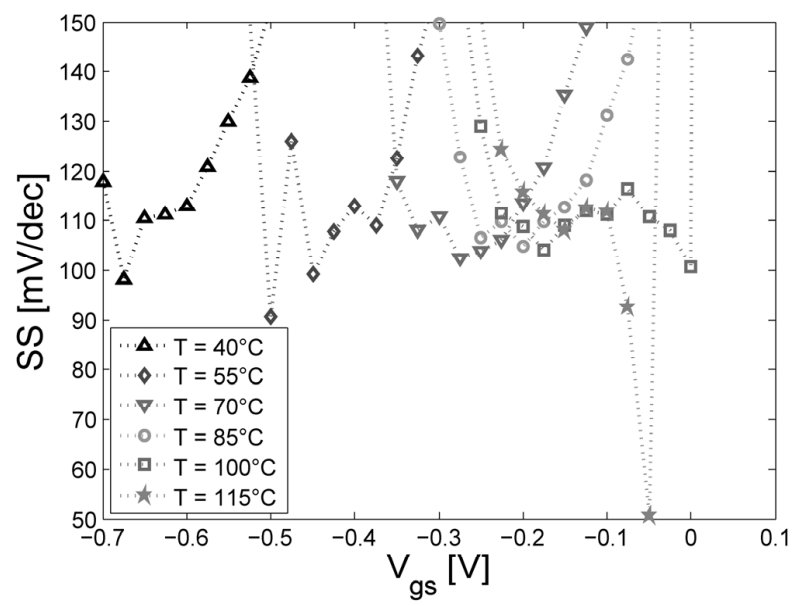

Figure 4: Subthreshold swings associated with the $I_{d s}-V_{g s}$ plots from Figure 3. Very low swing minima are measured at $100^{\circ} \mathrm{C}$ and $115^{\circ} \mathrm{C}$ close to threshold voltages. Notice the voltage shift with temperature increase and the extremely low minima of $50 \mathrm{mV} / \mathrm{dec}$ for the highest temperature.

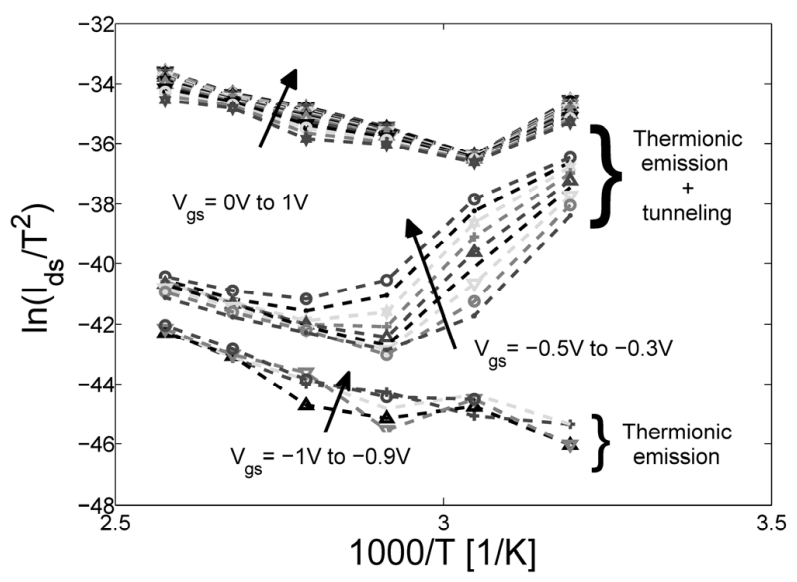

Figure 5: Arrhenius plot for different $V_{g s}$ extracted from the plots of Figure 3 showing both thermionic emission and tunneling mechanisms. The linear decreasing slopes are associated with thermionic emission regimes.

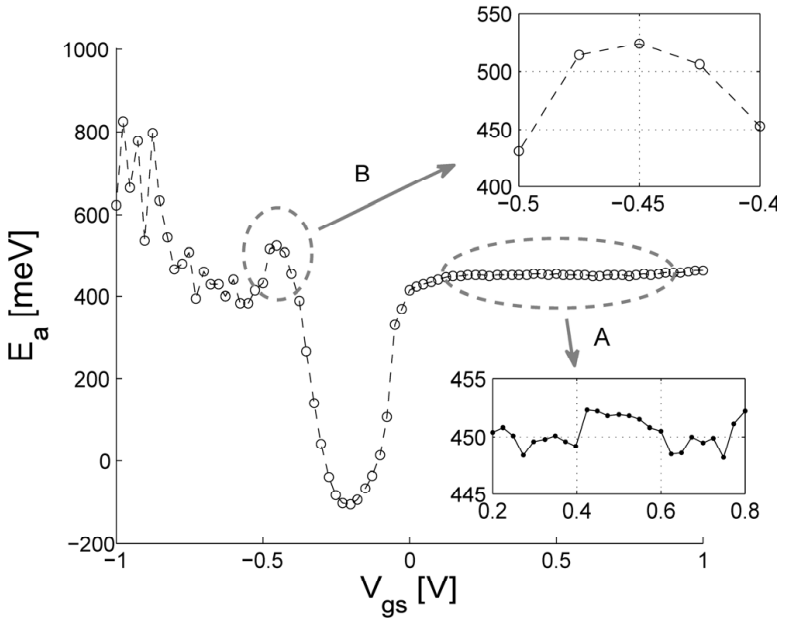

Figure 6: Extracted $E_{a}$ over a large range of $V_{g s}$. The inset $A$ shows a constant $E_{a}=450 \pm 5 \mathrm{meV}$ over $-0.2 \mathrm{~V} \leq V_{g s} \leq-0.8 \mathrm{~V}$. Inset $B$ shows the maximum of $E_{a}=525 \mathrm{meV}$ which is associated to the $\varphi_{B \text { Beff }}$

\section{SILICON NANOWIRE SCHOTTKY BARRIER FET FOR SENSING}

\section{pA current sensing}

Current biasing the devices with a constant $\mathrm{I}_{\mathrm{ds}}$ current makes the device to behave as a pseudo-inverter configuration with hysteretic transfer function. Thanks to the ambipolarity, the $\mathrm{V}_{\text {out }}-\mathrm{V}_{\text {in }}$ curves shift linearly with the applied current bias. For instance in Figure 7, low pA current levels can be either read from the high-to-low or the low-to-high transition voltage with sensitivities of 17 $\mathrm{mV} / \mathrm{pA}$. A similar biasing scheme for polysilicon nanowires has been previously characterized by the authors show a similar trend. In Figure 8, forward and reverse threshold voltages for currents between $100 \mathrm{fA}$ and $500 \mathrm{fA}$ show a linear increase with current (adapted from [8]).

\section{Temperature sensing}

Another application is temperature sensing. Upon application of increasing temperature of operation, the hysteresis window observed in pseudo-inverter biasing scheme shrinks.

The crystalline Si nanowire Schottky barrier FET shows different sensitivities at different temperature regimes, depending on which mechanism dominates the conductance. Since the hysteresis is attributed to the storage of charges in either gate oxide and/or at the Schottky barrier junctions [9], an increased hysteresis window is expected for the lowest temperatures. The highest sensitivity of $40 \mathrm{mV} /{ }^{\circ} \mathrm{C}$ is found in the $\mathrm{T}$ range around $40^{\circ} \mathrm{C}$ at which the trap tunneling mechanisms dominates. For temperatures higher than $55^{\circ} \mathrm{C}$ the sensitivity tends to saturate according to the dominance of thermionic current contribution, leading to lower sensitivity of $10 \mathrm{mV} /{ }^{\circ} \mathrm{C}$. In Figure 9 the hysteresis window shrink for increasing $\mathrm{T}$ when $70^{\circ} \mathrm{C} \leq \mathrm{T} \leq 100^{\circ} \mathrm{C}$ is shown. 


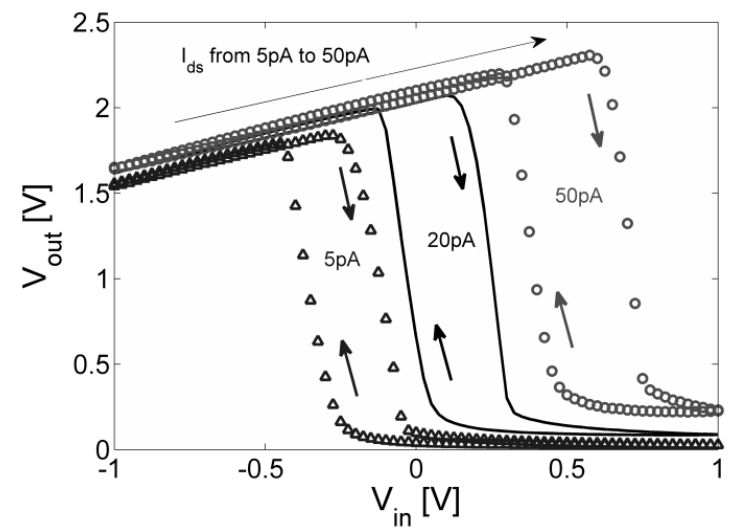

Figure 7: Measured input-output transfer characteristics of a hysteretic inverter based on a single Si nanowire FET with low current bias, showing current-dependent thresholds.
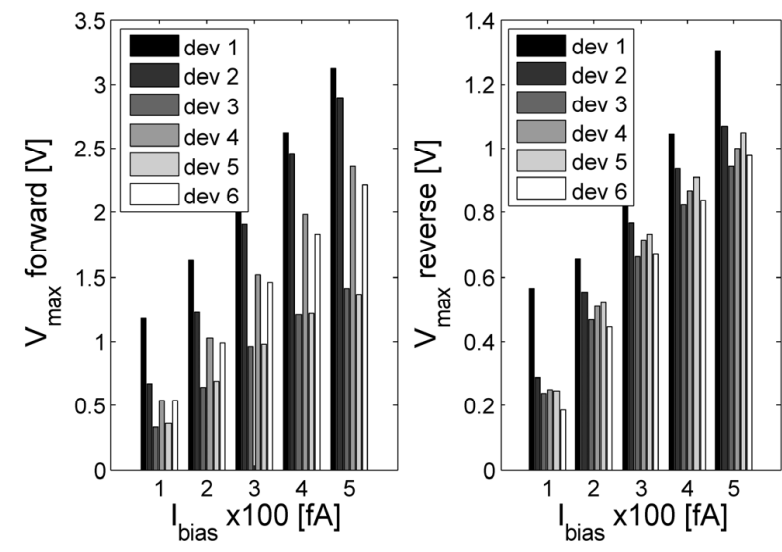

Figure 8: Forward and reverse threshold voltages for polysilicon Schottky barrier FETs under constant current biasing from $100 \mathrm{fA}$ up to $500 \mathrm{fA}$ (adapted from ref. [8]).

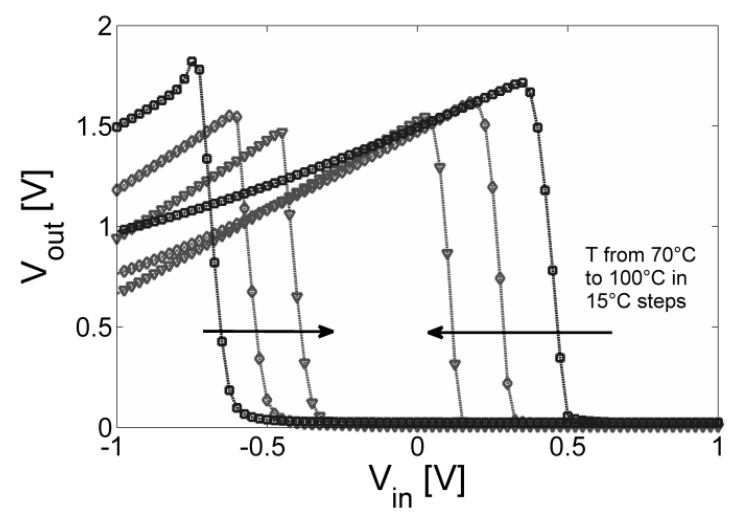

Figure 9: The hysteresis window shrinks with increasing temperature. Within this T range, the temperature sensitivity of $10 \mathrm{mV} /{ }^{\circ} \mathrm{C}$ is related with the thermionic current regime.

\section{CONCLUSIONS}

The Schottky barrier Si nanowire FET has been demonstrated suitable for precise current and temperature sensing. Sensitivities ranging in between 17 $\mathrm{mV} / \mathrm{pA}$ and $40 \mathrm{mV} / \mathrm{pA}$ for current sensing and in between $10 \mathrm{mV} /{ }^{\circ} \mathrm{C}$ and $40 \mathrm{mV} /{ }^{\circ} \mathrm{C}$ for temperature sensing associated with the different current conductance mechanisms are reported. The compatibility of $\mathrm{Si}$ nanowires for both sensing and logic applications makes these structures suitable for heterogeneous integration within the same fabrication platform scheme.

\section{ACKNOWLEDGEMENTS}

This works has been partially founded by the Swiss NSF Grant No 200021-122168, Swiss NSF Grant No 200021-132539. The authors would like to acknowledge Prof. Ali Shakouri of UCSC and Michael Zervas for useful discussion and the Center of Micro and Nanotechnology of EPFL for help with the fabrication.

\section{REFERENCES}

[1] H. Iwai, "Roadmap for $22 \mathrm{~nm}$ and beyond", Microelectronic Engineering, vol.86(7-9), 2009.

[2] F. Patolsky and C.M. Lieber, "Nanowire nanosensors," Materials Today, vol.8(4), pp.20-28, 2005.

[3] D. Sacchetto, M. Ben-Jamaa, G. De Micheli, and Y. Leblebici, "Fabrication and Characterization of Vertically Stacked Gate-all-Around Si Nanowire FET Arrays", Solid State Device Research Conference, 2009. ESSDERC '09. Proceedings of the European, 2009, pp. 245-248.

[4] M. Jang, K. Kang, S. Lee, and K. Park, "Simulation of Schottky barrier tunnel transistor using simple boundary condition," Applied Physics Letters, vol.82(16), pp.2718-2720, 2003.

[5] Y.-M. Lin, J. Appenzeller, J. Knoch, and P. Avouris, "High performance carbon nanotube field-effect transistor with tunable polarities", Nanotechnology, IEEE Transactions on, vol. 4, no. 5, pp. 481-489, Sept. 2005.

[6] S. M. Sze and K. K. Ng, "Physics of Semiconductor Devices", $3^{\text {rd }}$ edition, Jon Wiley and Sons, 2007.

[7] J. Svensson et al., "The dependence of the Schottky barrier height on carbon nanotube diameter for Pdcarbon nanotube contacts", Nanotechnology, vol. 20(17), pp. 175 204-+, 2009.

[8] D.Sacchetto, V.Savu, G. De Micheli, J. Brügger, Y. Leblebici, Ambipolar silicon nanowire FETs with stenciled-deposited metal gate, article in Press, Microelectronics Engineering, 2011.

[9] D.Sacchetto, M.H. Ben-Jamaa, S. Carrara, G. De Micheli, Y. Leblebici, Memristive Devices Fabricated with Silicon Nanowire Schottky barrier Transistors, Circuits and Systems (ISCAS) Proceedings of 2010 IEEE International Symposium on, 2010, pp. 9-12.

\section{CONTACT}

Davide Sacchetto

Tel: +41 216934698

davide.sacchetto@epfl.ch 